\title{
Balanced Parentheses Strike Back
}

\author{
HSUEH-I LU AND CHIA-CHI YEH
}

National Taiwan University

\begin{abstract}
An ordinal tree is an arbitrary rooted tree where the children of each node are ordered. Succinct representations for ordinal trees with efficient query support have been extensively studied. The best previously known result is due to Geary et al. [2004b, pages 1-10]. The number of bits required by their representation for an $n$-node ordinal tree $T$ is $2 n+o(n)$, whose first-order term is information-theoretically optimal. Their representation supports a large set of $O(1)$-time queries on $T$. Based upon a balanced string of $2 n$ parentheses, we give an improved $2 n+o(n)$-bit representation for $T$. Our improvement is two-fold: First, the set of $O(1)$-time queries supported by our representation is a proper superset of that supported by the representation of Geary, Raman, and Raman. Second, it is also much easier for our representation to support new queries by simply adding new auxiliary strings.

Categories and Subject Descriptors: E.1 [Data Structure]: -Trees; E.4 [Coding and Information Theory]: -Data compaction and compression; F.2.2 [Analysis of Algorithms and Problem Complexity]: Nonnumerical Algorithms and Problems-Computations on discrete structures; G.2.2 [Discrete Mathematics]: Graph Theory—Graph algorithms, Trees; H.3.1 [Information Storage and Retrieval]: Content Analysis and Indexing-Dictionaries, indexing methods
\end{abstract}

General Terms: Algorithm, Design, Theory

Additional Key Words and Phrases: Succinct data structures, XML document representation

ACM Reference Format:

Lu, H.-I., and Yeh, C.-C. 2008. Balanced parentheses strike back. ACM Trans. Algor. 4, 3, Article 28, (June 2008), 13 pages. DOI $=10.1145 / 1367064.1367068 \mathrm{http}: / /$ doi.acm.org/10.1145/ 1367064.1367068

\section{Introduction}

An ordinal tree (see, e.g., Geary et al. [2004b] and Benoit et al. [2005]) is an arbitrary rooted tree where the children of each node are ordered. All trees in this

This research was supported in part by NSC Grants 94-2213-F-002-126, 95-2221-E-002-077, and 96-2221-E-002-033.

H.-I. $\mathrm{Lu}$ is the corresponding author and is also affiliated with the Graduate Institute of Networking and Multimedia and the Graduate Institute of Biomedical Electronics and Bioinformatics, National Taiwan University.

Authors' address: Department of Computer Science and Information Engineering, National Taiwan University, 1 Roosevelt Road, Section 4, Taipei 106, Taiwan, Republic of China, e-mail: \{hil; r93048\}@csie.ntu.edu.tw.

Permission to make digital or hard copies of part or all of this work for personal or classroom use is granted without fee provided that copies are not made or distributed for profit or direct commercial advantage and that copies show this notice on the first page or initial screen of a display along with the full citation. Copyrights for components of this work owned by others than ACM must be honored. Abstracting with credit is permitted. To copy otherwise, to republish, to post on servers, to redistribute to lists, or to use any component of this work in other works requires prior specific permission and/or a fee. Permissions may be requested from Publications Dept., ACM, Inc., 2 Penn Plaza, Suite 701, New York, NY 10121-0701 USA, fax +1 (212) 869-0481, or permissions@acm.org.

(C) 2008 ACM 1549-6325/2008/06-ART28 \$5.00 DOI 10.1145/1367064.1367068 http://doi.acm.org/ $10.1145 / 1367064.1367068$ 


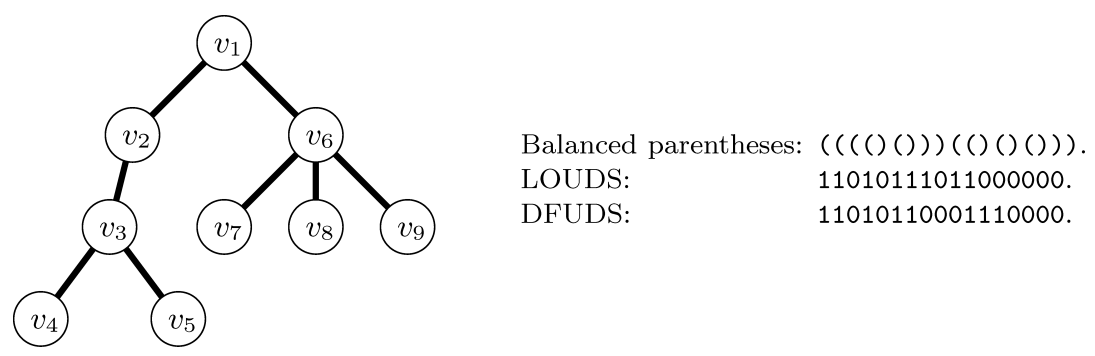

FIG. 1. Three representations for the same tree.

article are ordinal. The number of distinct $n$-node trees is $2^{2 n-\Theta(\log n)}$ [Graham et al. 1989], so the information-theoretically minimum number of bits to differentiate these trees is $2 n-\Theta(\log n)$. There are three major types of $2 n$-bit representations for an $n$-node tree $T$ :

—Balanced parentheses [Munro and Raman 2001; Chuang et al. 1998; He et al. 1999; Chiang et al. 2005; Munro and Rao 2004; Bonichon et al. 2006], a folklore encoding consisting of a balanced string of parentheses representing the counterclockwise depth-first traversal of $T$, where an open (respectively, closed) parenthesis denotes a descending (respectively, ascending) edge traversal. For technical reason, one usually adds a pair of enclosing parentheses to the above $2 n-2$ parentheses, resulting in a representation consisting of $2 n$ parentheses.

-Level order unary degree sequence (LOUDS) [Jacobson 1989], representing a node of degree $d$ as a string of $d$ copies of 1-bits followed by a 0 -bit, where these nodes are represented in a level-order traversal of $T$.

—Depth first unary degree sequence (DFUDS) [Benoit et al. 2005], representing a node of degree $d$ as a string of $d$ copies of 1-bits followed by a 0-bit, where these nodes are represented in a depth-first traversal of $T$.

An example is shown in Figure 1.

Initiated by Jacobson [1989], succinct representations for trees with efficient query support have been extensively studied in the literature. Jacobson [1989] extended the LOUDS representation into a $\Theta(n)$-bit encoding to support the parent query and the rank and select queries for nodes in level-order traversal of $T$ in $\Theta(\log n)$ time. Clark and Munro [Clark 1996; Clark and Munro 1996] squeezed Jacobson's encoding into a $3 n+o(n)$-bit representation, from which the above queries and the subtree-size query can be supported in $O(1)$ time. Later succinct representations, all have $2 n+o(n)$ bits, form the following trade-off between the choices of base representations and the sets of supported $O(1)$-time queries:

-Based upon balanced parentheses, Munro and Raman [2001] showed that an $o(n)$-bit auxiliary string suffices to support the following queries in $O(1)$ time: parent, depth, subtree-size, and the rank and select queries for nodes in pre-order and post-order traversal of $T$. Munro et al. [2001] showed an $o(n)$-bit auxiliary string to support $O(1)$-time query for leaf-rank, leaf-select, and leaf-size. Chiang et al. [2005] showed an $o(n)$-bit auxiliary string to support $O(1)$-time degree query. Munro and Rao [2004] further gave an $o(n)$-bit auxiliary string to support $O$ (1)-time level-ancestor query. 
TABLE I. A SUMMARY FOR CURRENT $2 n+o(n)$-BIT ENCODINGS FOR AN $n$-NODE TREE: PARENTHESES [MunRo AND RAMAN 2001, 2004; ChiANG ET AL. 2005; MunRo AND RAO 2004; MunRo ET AL. 2001], DFUDS [BENOIT ET AL. 2005], GEARY ET AL. [2004a]

\begin{tabular}{|l||c|c|c|c|}
\hline & Parentheses & DFUDS & Geary et al. & new \\
\hline \hline pre-order select and rank & $\vee$ & $\vee$ & $\vee$ & $\vee$ \\
\hline post-order select and rank & $\vee$ & & $\vee$ & $\vee$ \\
\hline child-select and child-rank & & $\vee$ & $\vee$ & $\vee$ \\
\hline leaf-select, leaf-rank, and leaf-size & $\vee$ & & & $\vee$ \\
\hline lowest common ancestor & & & & $\vee$ \\
\hline subtree height & & & & $\vee$ \\
\hline subtree size & $\vee$ & $\vee$ & $\vee$ & $\vee$ \\
\hline level ancestor & $\vee$ & & $\vee$ & $\vee$ \\
\hline distance & & & & $\vee$ \\
\hline degree & $\vee$ & $\vee$ & $\vee$ & $\vee$ \\
\hline depth & $\vee$ & & $\vee$ & $\vee$ \\
\hline
\end{tabular}

-Based upon the DFUDS representation, Benoit et al. [2005] gave an $o(n)$-bit auxiliary string that supports the following queries in $O(1)$ time: child-rank, child-select, degree, subtree-size, and node-rank and node-select in the pre-order traversal of $T$. However, such a choice of the base representation still does not provide $O(1)$-time support for the depth and level-ancestor queries, the noderank and node-select queries in the post-order traversal of $T$, and the rank, select, and size queries for leaves.

Recently, Geary et al. [2004b] almost resolved the above trade-off by giving a $2 n+o(n)$-bit encoding for $T$ that supports in $O(1)$ time the aforementioned queries except those leaf-related ones [Munro et al. 2001]. Their approach differs from all previous work achieving $2 n+o(n)$ bits in that their encoding does not consist of a $2 n$-bit base representation for the topology of $T$ plus an $o(n)$-bit auxiliary string. Instead, they decomposed $T$ into several types of subtrees, whose topologies are represented in a hierarchical way, where different levels are composed of mixtures of different base representations and auxiliary strings. Such an involved structure seriously complicates the possibility of supporting additional queries using other stand-alone auxiliary strings. An implementation based upon a similar concept is studied in Geary et al. [2004b]. Very recently, Delpratt et al. [2006] showed that LOUDS-based representation can also be implemented to have competitive practical performance.

In this article, we give new $o(n)$-bit auxiliary strings for the $2 n$-bit balanced string of parentheses representing $T$. Together with previous $o(n)$-bit auxiliary strings for balanced parentheses [Munro and Raman 2001; Chiang et al. 2005; Munro and Rao 2004], our $2 n+o(n)$-bit encoding for $T$ supports all of Geary et al.'s queries in $O(1)$ time. Consisting of a base representation plus $o(n)$-bit auxiliary strings, our encoding is better in the ease of supporting new queries by adding new $o(n)$-bit auxiliary strings. To demonstrate such an advantage, we also show how to handle $O$ (1)-time queries currently unsupported by Geary et al.'s encoding, including (a) lowest common ancestor, (b) distance, and (c) subtree height. Table I summarizes the above discussion.

We follow the convention of unit-cost RAM model of computation with $\Theta(\log n)$ bit word size [van Emde Boas 1990], which is assumed in all the previous work except that of Jacobson [1989]. The rest of this article is organized as follows. 
Section 2 gives the preliminaries. Section 3 shows our auxiliary strings for distance, subtree height, and lowest common ancestor. Section 4 shows our auxiliary strings for child-rank and child-select.

\section{Preliminaries}

Let $T$ be the input $n$-node tree. Let $v_{i}$ denote the $i$ th node of $T$ in the pre-order traversal of $T$. Let $S$ be the balanced string of $2 n$ parentheses for $T$. Let $S[i, j]$ denote the substring of $S$ from index $i$ to index $j$. Let $S[i]=S[i, i]$. Let $\ell_{i}$ be the index such that $S\left[\ell_{i}\right]$ is the $i$-th open parenthesis in $S$. Let $r_{i}$ be the index such that $S\left[r_{i}\right]$ is the closed parenthesis that matches $S\left[\ell_{i}\right]$ in $S$. One can easily see that the correspondence between $v_{i}$ and the matched parentheses $S\left[\ell_{i}\right]$ and $S\left[r_{i}\right]: v_{i}$ is the parent of $v_{j}$ if and only if $S\left[\ell_{i}\right]$ and $S\left[r_{i}\right]$ is the closest parenthesis pair that encloses $S\left[\ell_{j}\right]$ and $S\left[r_{j}\right]$. Let $w(i, j)=j-i+1$. For the rest of the paper, all logarithms are of base 2. Let $B=\left\lceil\log ^{3} n\right\rceil, b=\left\lceil(\log \log n)^{3}\right\rceil, n_{B}=\left\lceil\frac{2 n}{B}\right\rceil$, and $n_{b}=\left\lceil\frac{2 n}{b}\right\rceil$.

LEMMA 2.1 (SEE Bell ET AL. [1990] AND Elias [1975]). For any $O(n)$-bit strings $S_{1}, S_{2}, \ldots, S_{k}$ with $k=O(1)$, there is an $O(\log n)$-bit auxiliary string $\alpha_{\text {concat }}$ such that, given the concatenation of $\alpha_{\text {concat }}, S_{1}, S_{2}, \ldots, S_{k}$ as input, the index of the first symbol of any given $S_{i}$ in the concatenation is computable in $O(1)$ time.

Let $S_{1} \circ S_{2} \circ \cdots \circ S_{k}$ denote the concatenation of $\alpha_{\text {concat }}, S_{1}, S_{2}, \ldots, S_{k}$ as in Lemma 2.1.

LEMMA 2.2 (SEE MUNRO AND RAMAN [2001] AND CHIANG ET AL. [2005]). Let $S$ be a length- $2 n$ string of balanced parentheses that represents an $n$-node tree $T$. It takes $O(n)$ time to compute an o(n)-bit string $\alpha_{\text {aux }}$ such that the following queries for $S$ can be determined from $S$ and $\alpha_{\text {aux }}$ in $O(1)$ time: (a) the parent, degree, and depth of $v_{i}$ in $T$, (b) the parenthesis that matches $S[i]$ in $S$, and $(c)$ the rank and select queries for open and closed parentheses in $S$.

By Lemma 2.2, given $S \circ \alpha_{\text {aux }}$, indices $i, \ell_{i}$, and $r_{i}$ can be determined from one another in $O(1)$ time. Our technique of dividing the input strings into multiple levels of blocks, which has been widely used in many succinct data structures, is inspired by Munro and Raman [Munro 1996; Munro and Raman 2001].

\section{Distance, Subtree Height, and Lowest Common Ancestor}

Let $L$ be the $2 n$-element array such that each $L[i]$ is the number of open parentheses minus the number of closed parentheses in $S[1, i]$. Therefore, if $S[j]$ is the $i$ th open parenthesis in $S$, then $L[j]$ is the level of $v_{i}$ in $T$. For any indices $i$ and $j$ with $i \leq j$, let $\operatorname{index}_{\min }(L, i, j)$ (respectively, $\operatorname{index}_{\max }(L, i, j)$ ) denote the smallest index $k$ with $i \leq k \leq j$ such that $L[k]$ equals the minimum (respectively, maximum) of $L[i], L[i+1], \ldots, L[j]$. As observed by Gabow et al. [1984], the lowest-common-ancestor query can be reduced to the above

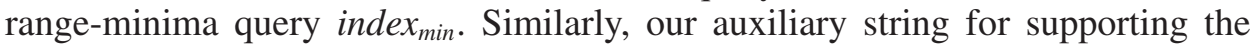
queries of distance, subtree height, and lowest common ancestor is based on the lemma below. Observe that each $L[i]$ can be obtained from $S$ in $O(1)$ time using the auxiliary string $\alpha_{a u x}$ for the rank queries with respect to open and 
closed parentheses in $S$. Therefore, the following lemma does not require $L$ in the encoding.

Let $I$ be an array of $m$ indices. Let $k_{\min }(I, m, i, j)$ (respectively, $k_{\max }(I, m, i, j)$ ) be the smallest index $k$ with $i \leq k \leq j$ that minimizes (respectively, maximizes) $L[I[k]]$. We first prove the following lemma using techniques extended from Section 3 of Bender and Farach-Colton [2000].

LEMMA 3.1. It takes $O(m \log m)$ time to compute an $O\left(m \log ^{2} m\right)$-bit string $\alpha_{q}(I, m)$ from which $k_{\min }(I, m, i, j)$ and $k_{\max }(I, m, i, j)$ for any indices $i$ and $j$ with $1 \leq i \leq j \leq m$ can be determined from $S, \alpha_{\text {aux }}$, and $\alpha_{q}$ in $O(1)$ time.

PROOF. For each $i=1,2, \ldots, m$ and $j=1,2, \ldots,\lceil\log m\rceil$, let $M_{\min }[i][j]$ (respectively, $M_{m}[i][j]$ ) be the smallest index $k$ with $i \leq k<i+2^{j}$ that minimizes (respectively, maximizes) $L[I[k]]$. Let $\alpha_{q}(I, m)=M_{\min } \circ M_{\max }$. Observe that $\alpha_{q}(I, m)$ takes $O\left(m \log ^{2} m\right)$ bits and can be computed from $L$ and $I$ in $O(m \log m)$ time using dynamic programming. Let $k_{1}=M_{\min }[i][k]$ and $k_{2}=M_{\min }\left[j-2^{k}+1\right][k]$, where $k=\lfloor\log (j-i)\rfloor$. It is not difficult to see that

$$
k_{\text {min }}(I, m, i, j)=\left\{\begin{array}{l}
k_{1} \text { if } L\left[I\left[k_{1}\right]\right]<L\left[I\left[k_{2}\right]\right] \\
k_{2} \text { otherwise. }
\end{array}\right.
$$

One can compute $k_{\max }(I, m, i, j)$ from $M_{\max }, I$, and $L$ analogously in $O(1)$ time.

LEMMA 3.2. It takes $O(n)$ time to compute an o(n)-bit string $\alpha_{r m q}$ such that index $_{\min }(L, i, j)$ and index $x_{\max }(L, i, j)$ for any indices $i$ and $j$ can be computed from $S$, $\alpha_{\text {aux }}$, and $\alpha_{r m q}$ in $O(1)$ time.

PROOF. First let $I_{B}$ be the $n_{B}$-element array such that each $I_{B}[i]$ is the smallest index $j$ with $(i-1) B<j \leq i B$ that minimizes $L[j]$. $I_{B}$ takes $O\left(n_{B} \log B\right)=o(n)$ bits. Also, for each $i=1,2, \ldots, n_{B}$, let $I_{b}[i]$ be the $\left\lceil\frac{B}{b}\right\rceil$-element array such that each $I_{b}[i][j]$ is the smallest index $t$ with $(j-1) b<t \leq j b$ that minimizes $L[(i-1) B+t] . I_{b}$ takes $O\left(n_{B}\left\lceil\frac{B}{b}\right\rceil \log b\right)=o(n)$ bits. Let $\alpha_{q 1}=\alpha_{q}\left(I_{B}, n_{B}\right)$, and for each $i=1,2, \ldots, n_{B}$, let $\alpha_{q 2}[i]=\alpha_{q}\left(I_{b}[i],\left\lceil\frac{B}{b}\right\rceil\right)$. By Lemma 3.1, both of $\alpha_{q 1}$ and $\alpha_{q 2}$ take $o(n)$ bits and can be obtained in $O(n)$ time. Finally, let $\alpha_{q 3}$ be an $O(n)$-time obtainable table such that any $\operatorname{index}_{\min }(L, i, j)$ and $\operatorname{index}_{\max }(L, i, j)$ with $w(i, j) \leq 2 b$ can be computed from $S[i, j]$ and $\alpha_{q 3}$ in $O(1)$ time. That is, let $\alpha_{q 3}[S[i, i+2 b-1]][j-i+1]=\left(\operatorname{index}_{\min }(L, i, j)-i\right.$, index $\left._{\max }(L, i, j)-i\right)$ for any indices $i$ and $j$ with $w(i, j) \leq 2 b$. Since each entry takes $O(\log b)$ bits, the number of bits required by $\alpha_{q 3}$ is $O\left(2^{2 b} 2 b \log b\right)=o(n)$. Let $\alpha_{r m q}=\alpha_{q 1} \circ \alpha_{q 2} \circ \alpha_{q 3} \circ I_{B} \circ I_{b}$, which has $o(n)$ bits and is obtainable in $O(n)$ time.

To answer $\operatorname{index}_{\min }(L, i, j)$ from $S, \alpha_{\text {aux }}$, and $\alpha_{r m q}$, we can always decompose the interval $[i, j]$ into two (not necessarily disjoint) subintervals $\left[i_{1}, j_{1}\right]$ and $\left[i_{2}, j_{2}\right]$ whose union is $[i, j]$. Clearly $\operatorname{index}_{\min }(L, i, j)$ can be determined from index $_{\min }\left(L, i_{1}, j_{1}\right)$ and index $\min _{\text {m }}\left(L, i_{2}, j_{2}\right)$ in $O(1)$ time. Consider the following cases.

- Case 1. $w(i, j) \leq 2 b$. We simply resort to $S[i, j]$ and $\alpha_{q 3}$.

- Case 2. $w(i, j)>2 b$ and $S[i, j]$ is in the same length- $B$ block of $S$. Since index $_{\text {min }}(L, i, i+b-1)$ and index $x_{\text {min }}(L, j-b+1, j)$ can be determined in $O(1)$ time using Case 1 , it suffices to determine $\operatorname{index}_{\min }\left(L, i^{\prime}, j^{\prime}\right)$, where (a) $i^{\prime}$ is the smallest index with $i \leq i^{\prime}$ that is a starting index of a length- $b$ block of $S$, and (b) $j^{\prime}$ is the largest index with $j^{\prime} \leq j$ that is an ending index of a length- $b$ block 
of $S$. Since $i^{\prime}$ and $j^{\prime}$ are in the same length- $B$ block of $S$, index $\min _{\text {min }}\left(L, i^{\prime}, j^{\prime}\right)$ can be determined from $S, \alpha_{a u x}$, and $\alpha_{q 2}$ in $O(1)$ time.

- Case 3. $w(i, j)>2 b$ and $S[i, j]$ belongs to two or more consecutive length- $B$ blocks of $S$. Let $i^{\prime}-1$ be the ending index of the length- $B$ block of $S$ that contains $i$. Let $j^{\prime}+1$ be the starting index of the length- $B$ block of $S$ that contains $j$. Since $\operatorname{index}_{\min }\left(L, i, i^{\prime}-1\right)$ and $\operatorname{index}_{\min }\left(L, j^{\prime}+1, j\right)$ can be determined in $O(1)$ time using Case 2, it suffices to determine index $\min _{\min }\left(L, i^{\prime}, j^{\prime}\right)$ for the case that $i^{\prime} \leq j^{\prime}$. Since $i^{\prime}$ is a starting index of a length- $B$ block of $S$ and $j^{\prime}$ is an ending index of a length- $B$ block of $S$, one can determine $\operatorname{index}_{\min }\left(L, i^{\prime}, j^{\prime}\right)$ from $S, \alpha_{a u x}$, and $\alpha_{q 1}$ in $O(1)$ time.

It is not difficult to answer $\operatorname{index}_{\max }(L, i, j)$ from $S, \alpha_{\text {aux }}$, and $\alpha_{r m q}$ analogously in $O(1)$ time.

As pointed out by an anonymous reviewer, our data structure for lowest common ancestor is similar to that of Sadakane [2002] for suffix arrays.

THEOREM 3.3. It takes $O(n)$ time to compute an o(n)-bit string $\alpha_{\text {new } 1}$ such that the queries of distance, subtree height, and lowest common ancestor can be answered from $S$ and $\alpha_{\text {new } 1}$ in $O(1)$ time.

PROOF. Let $\alpha_{\text {new } 1}=\alpha_{\text {aux }} \circ \alpha_{r m q}$. By Lemmas 2.2 and 3.2, $\alpha_{\text {new } 1}$ has $o(n)$ bits and can be computed from $S$ in $O(n)$ time.

- The height of the subtree rooted at $v_{i}$ is $L\left[\operatorname{index}_{\max }\left(L, \ell_{i}, r_{i}\right)\right]$ minus the depth of $v_{i}$ in $T$.

-The lowest common ancestor $v_{k}$ of $v_{i}$ and $v_{j}$ with $\ell_{i}<\ell_{j}$ can be determined as follows. If $r_{i}>r_{j}$, then $v_{k}=v_{i}$. Otherwise, $S\left[\operatorname{index}_{\min }\left(L, r_{i}, \ell_{j}\right)\right]$ has to be a closed parenthesis $r_{x}$ such that $v_{x}$ is a child of $v_{k}$, as observed by Bender and Farach-Colton [2000].

-The distance of $v_{i}$ and $v_{j}$ is exactly the depth of $v_{i}$ plus the depth of $v_{j}$ minus two times of the depth of $v_{k}$, where $v_{k}$ is the lowest common ancestor of $v_{i}$ and $v_{j}$.

By Lemmas 2.2 and 3.2, the above queries can all be answered from $S$ and $\alpha_{\text {new } 1}$ in $O(1)$ time.

\section{Rank and Select for Children}

Before solving rank and select for children, we introduce the following definition and its property. A non-root node $v_{i}$ is $k$-far if $w\left(\ell_{p}, \ell_{i}\right)>k$ and $w\left(\ell_{i}, r_{p}\right)>k$, where $v_{p}$ is the parent of $v_{i}$.

LEMMA 4.1. If $v_{i}$ and $v_{j}$ are two $k$-far non-root nodes with $\left|w\left(\ell_{i}, \ell_{j}\right)\right| \leq k$, then $v_{i}$ and $v_{j}$ are siblings.

ProOF. Without loss of generality, we assume $\ell_{i}<\ell_{j}$. Since $v_{i}$ and $v_{j}$ are $k$-far non-root nodes with $w\left(\ell_{i}, \ell_{j}\right) \leq k, v_{i}$ cannot be an ancestor or descendant of $v_{j}$. Thus, we have $r_{i}<\ell_{j}$. Assume for a contradiction that $v_{p}$ (respectively, $v_{q}$ ) is the parent of $v_{i}$ (respectively, $v_{j}$ ) and $v_{p} \neq v_{q}$. Observe that either $r_{i}<\ell_{q}$ or $r_{p}<\ell_{j}$ holds. Since $v_{j}$ is $k$-far, $r_{i}<\ell_{q}$ implies $w\left(r_{i}, \ell_{j}\right)>k$. Since $v_{i}$ is $k$-far, 
$r_{p}<\ell_{j}$ implies $w\left(r_{i}, \ell_{j}\right)>k$. Either case leads to a contradiction, so the lemma is proved.

For presentational brevity, we classify non-root nodes into the following three disjoint classes: A node is

-narrow if it is not $b$-far;

- medium if it is $b$-far but not $B$-far; and

—wide if it is $B$-far.

4.1. ChILD RANK. Let child_rank $\left(S, v_{k}\right)$ denote the number $c$ such that $v_{k}$ is the $c$ th child of its parent. We have the following theorem.

THEOREM 4.2. It takes $O(n)$ time to compute an o(n)-bit string $\alpha_{\text {new } 2}$ such that child_rank $\left(S, v_{k}\right)$ for each node $v_{k}$ can be answered from $S$ and $\alpha_{\text {new } 2}$ in $O(1)$ time.

PROOF. Let $v_{p}$ be the parent of $v_{k}$. If $S[i, j]$ is a balanced string of parentheses, let $\operatorname{sibling}(S, i, j)$ be the number of non-enclosed parenthesis pairs in $S[i, j]$. Observe that

$$
\begin{aligned}
& \operatorname{child} \operatorname{rank}\left(S, v_{k}\right)=\operatorname{sibling}\left(S, \ell_{p}+1, \ell_{k}-1\right)+1 \\
& =\operatorname{degree}\left(S, v_{p}\right)-\operatorname{sibling}\left(S, \ell_{k}, r_{p}-1\right)+1 \text {. }
\end{aligned}
$$

Therefore, it remains to support each query $\operatorname{sibling}(S, i, j)$ in $O(1)$ time.

If $v_{k}$ is narrow, we only need to answer $\operatorname{sibling}(S, i, j)$ with $w(i, j) \leq b$. We simply build an $O(n)$-time obtainable table $M_{1}$ to store the answers for any possible inputs. That is, let $M_{1}[S[i, i+b-1]][j-i+1]=\operatorname{sibling}(S, i, j)$ for any indices $i$ and $j$ with $w(i, j) \leq b$. Since $\operatorname{sibling}(S, i, j) \leq w(i, j)$, each entry requires $O(\log b)$ bits and $M_{1}$ takes $O\left(2^{b} b \log b\right)=o(n)$ bits.

If $v_{k}$ is medium, we cannot afford to store all the answers of $\operatorname{sibling}(S, i, j)$ with $w(i, j) \leq B$. We split $S$ into length- $b$ blocks. By Lemma 4.1, any two medium nodes $v_{i}$ and $v_{j}$ with $\left|w\left(\ell_{i}, \ell_{j}\right)\right| \leq b$ have the same parent, so for each block we save at most one medium node as a shortcut. Define tables $M_{2}$ and $M_{3}$ as follows. For each $t=1,2, \ldots, n_{b}$,

-let $M_{2}[t]=\left(\ell_{i}, \operatorname{sibling}\left(S, \ell_{p}+1, \ell_{i}-1\right)\right)$, where $\ell_{i}$ is the smallest index, if any, with $(t-1) b<\ell_{i} \leq t b$ such that $v_{i}$ is a medium child of $v_{p}$ with $w\left(\ell_{p}, \ell_{i}\right) \leq B$; and

-let $M_{3}[t]=\left(\ell_{i}, \operatorname{sibling}\left(S, \ell_{i}, r_{p}-1\right)\right)$, where $\ell_{i}$ is the smallest index, if any, with $(t-1) b<\ell_{i} \leq t b$ such that $v_{i}$ is a medium child of $v_{p}$ with $w\left(\ell_{i}, r_{p}\right) \leq B$.

Note that $M_{2}$ and $M_{3}$ have $n_{b}$ entries, each requiring $O(\log B)$ bits, so both of them take $O\left(n_{b} \log B\right)=o(n)$ bits. Therefore, for any medium child $v_{k}$ of $v_{p}$, if $w\left(\ell_{p}, \ell_{k}\right) \leq B$, then

$$
\begin{aligned}
\operatorname{sibling}\left(S, \ell_{p}+1, \ell_{k}-1\right) & =\operatorname{sibling}\left(S, \ell_{p}+1, \ell_{i}-1\right)+\operatorname{sibling}\left(S, \ell_{i}, \ell_{k}-1\right) \\
& =m+M_{1}\left[S\left[\ell_{i}, \ell_{i}+b-1\right]\right]\left[\ell_{k}-\ell_{i}\right],
\end{aligned}
$$

where $\left(\ell_{i}, m\right)=M_{2}\left[\left\lceil\frac{\ell_{k}}{b}\right]\right]$. Similarly, if $w\left(\ell_{k}, r_{p}\right) \leq B$, then

$$
\begin{aligned}
\operatorname{sibling}\left(S, \ell_{k}, r_{p}-1\right) & =\operatorname{sibling}\left(S, \ell_{i}, r_{p}-1\right)-\operatorname{sibling}\left(S, \ell_{i}, \ell_{k}-1\right) \\
& =m-M_{1}\left[S\left[\ell_{i}, \ell_{i}+b-1\right]\right]\left[\ell_{k}-\ell_{i}\right],
\end{aligned}
$$

where $\left(\ell_{i}, m\right)=M_{3}\left[\left\lceil\frac{\ell_{k}}{b}\right\rceil\right]$. 


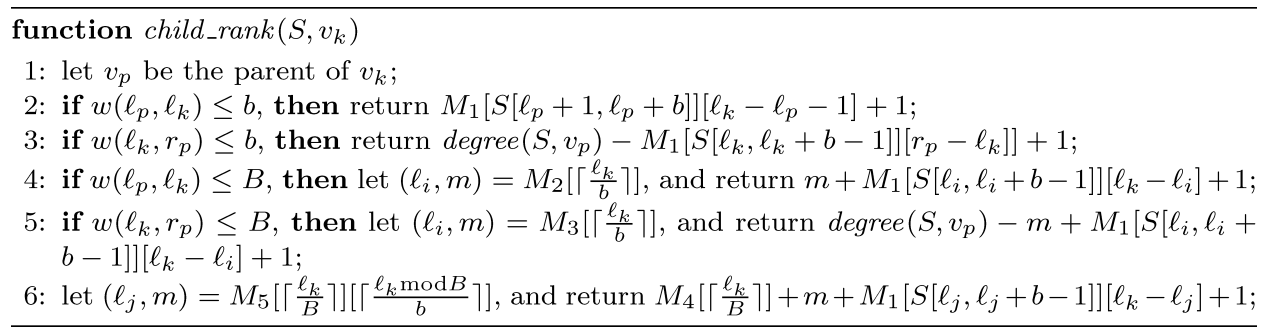

FIG. 2. An $O(1)$-time algorithm that computes $\operatorname{child} \_r a n k\left(S, v_{k}\right)$.

Similar tricks work for wide nodes, but they have to be applied in two levels. We first split $S$ into length- $B$ blocks. For each $t=1,2, \ldots, n_{B}$, let $M_{4}[t]=$ $\operatorname{sibling}\left(S, \ell_{p}+1, \ell_{i}-1\right)$, where $\ell_{i}$ is the smallest index, if any, with $(t-1) B<$ $\ell_{i} \leq t B$ such that $v_{i}$ is a wide child of $v_{p}$. We further split each length- $B$ block into length- $b$ blocks. For each $t=1,2, \ldots, n_{B}$ and $u=1,2, \ldots,\left\lceil\frac{B}{b}\right\rceil$, let $M_{5}[t][u]=$ $\left(\ell_{j}, \operatorname{sibling}\left(S, \ell_{p}+1, \ell_{j}-1\right)-M_{4}[t]\right)$, where $\ell_{j}$ is the smallest index, if any, with $(u-1) b<\ell_{j}-(t-1) B \leq u b$ such that $v_{j}$ is a wide child of $v_{p}$. Note that $\operatorname{sibling}\left(S, \ell_{p}+1, \ell_{j}-1\right)-M_{4}[t] \leq B$. One can easily verify that the number of bits required by $M_{4}$ is $O\left(n_{B} \log n\right)=o(n)$ and the number of bits required by $M_{5}$ is $O\left(n_{B}\left\lceil\frac{B}{b}\right\rceil \log B\right)=o(n)$. Thus, for any wide child $v_{k}$ of $v_{p}$, we have

$$
\begin{aligned}
\operatorname{sibling}\left(S, \ell_{p}+1, \ell_{k}-1\right) & =\operatorname{sibling}\left(S, \ell_{p}+1, \ell_{j}-1\right)+\operatorname{sibling}\left(S, \ell_{j}, \ell_{k}-1\right) \\
& =M_{4}\left[\left\lceil\frac{\ell_{k}}{B}\right\rceil\right]+m+M_{1}\left[S\left[\ell_{j}, \ell_{j}+b-1\right]\right]\left[\ell_{k}-\ell_{j}\right],
\end{aligned}
$$

where $\left(\ell_{j}, m\right)=M_{5}\left[\left\lceil\frac{\ell_{k}}{B}\right\rceil\right]\left\lceil\left\lceil\frac{\ell_{k} \bmod B}{b}\right\rceil\right]$.

Finally, let $\alpha_{\text {new } 2}=\alpha_{\text {aux }} \circ M_{1} \circ M_{2} \circ M_{3} \circ M_{4} \circ M_{5}$, which is an $o(n)$-bit string obtainable from $S$ in $O(n)$ time. The $O(1)$-time algorithm for computing child_rank $\left(S, v_{k}\right)$ is shown in Figure 2.

4.2. Child SELECT. First, we need the following lemmas to handle the select query for children. For any node $v_{i}$, let $\operatorname{index}_{c}\left(S, \ell_{i}, m, c\right)=\ell_{j}-\ell_{i}$, where $v_{j}$ is a sibling of $v_{i}$ with $w\left(\ell_{i}, \ell_{j}\right) \leq m$ such that child_rank $\left(S, v_{j}\right)=\operatorname{child} \_r a n k\left(S, v_{i}\right)+c$. If such a $v_{j}$ does not exist, $\operatorname{index}_{c}\left(S, \ell_{i}, m, c\right)=\phi$.

LEMMA 4.3. It takes $O(n)$ time to compute an o(n)-bit string $\alpha_{b}$ such that index $_{c}\left(S, \ell_{i}, b^{2}, c\right)$ for any node $v_{i}$ and index $c$ can be computed from $S$ and $\alpha_{b}$ in $O(1)$ time.

PROOF. We simply build an $O(n)$-time obtainable table $\alpha_{b}$ to store the answers for any possible inputs. That is, let $\alpha_{b}\left[S\left[\ell_{i}, \ell_{i}+b^{2}-1\right]\right][c]=\operatorname{index}_{c}\left(S, \ell_{i}, b^{2}, c\right)$ for any node $v_{i}$ and index $c$. Since each entry takes $O(\log b)$ bits, $\alpha_{b}$ requires $O\left(2^{b^{2}} b^{2} \log b\right)=o(n)$ bits.

LEMMA 4.4. Given a node $v_{i}$, it takes $O(B)$ time to compute an $o(B)$-bit string $\alpha_{B}\left(\ell_{i}\right)$ such that index $\left(S, \ell_{i}, B, c\right)$ for any index c can be computed from $S, \alpha_{b}$, and $\alpha_{B}\left(\ell_{i}\right)$ in $O(1)$ time.

PROOF. For each $t=0,1, \ldots,\left\lceil\frac{B}{b}\right\rceil-1$, let $W_{1}[t]=\operatorname{index}_{c}\left(S, \ell_{i}, B, t b\right) . W_{1}$ takes $O\left(\left\lceil\frac{B}{b}\right\rceil \log B\right)=o(B)$ bits. If $w\left(W_{1}[t], W_{1}[t+1]\right)>b^{2}$, we save the answers 
of $\operatorname{index}_{c}\left(S, \ell_{i}, B, t b+z\right)$ for each $z=0,1, \ldots, b-1$ in $W_{2} . W_{2}$ takes at most $O\left(\left\lceil\frac{B}{b^{2}}\right\rceil b \log B\right)=o(B)$ bits. Otherwise, by Lemma $4.3 \operatorname{index}_{c}\left(S, \ell_{i}, B, t b+z\right)$ can be computed in $O(1)$ time using $W_{1}[t]+\operatorname{index}_{c}\left(S, \ell_{i}+W_{1}[t], b^{2}, z\right)$. Let $\alpha_{B}\left(\ell_{i}\right)=W_{1} \circ W_{2}$, which has $o(B)$ bits and is obtainable in $O(B)$ time.

Given an array $A$ of $\left\lceil\frac{m}{u}\right\rceil$ positive $\lceil\log u\rceil$-bit integers with $m \leq n$ and $u=$ $\left\lceil\log ^{3} m\right\rceil$, let index $\operatorname{sum}_{\text {sum }}(A, x)$ denote the largest index $y$ with $\sum_{t=1}^{y} A[t]<x$.

LEMMA 4.5. It takes $O(m)$ time to compute an o(m)-bit string $\alpha_{A}(A, m)$ such that index $\operatorname{sum}(A, x)$ for any index $x$ can be determined from $A$ and $\alpha_{A}(A, m)$ in $O(1)$ time.

PROOF. This is a special case of the search query of the searchable partial sums problem [Raman et al. 2001; Hon et al. 2003]. Theorem 3 of Hon et al. [2003] gave an $o(m)$-bit auxiliary string to support this query in $O(1)$ time, but it is unclear whether the preprocessing time is $O(m)$. Let us briefly prove this lemma as follows:

Let $d\left(x_{1}, x_{2}\right)$ denote $\operatorname{index}_{\text {sum }}\left(A, x_{2}\right)-\operatorname{index}_{\text {sum }}\left(A, x_{1}\right)$. For each $t=$ $0, \ldots,\left\lceil\frac{m}{u}\right\rceil-1$, let $W_{3}[t]=\operatorname{index}_{\text {sum }}(A, t u) . W_{3}$ needs $O\left(\left\lceil\frac{m}{u}\right\rceil \log m\right)=o(m)$ bits. If $d(t u,(t+1) u)>\left\lceil\log ^{2} u\right\rceil$, for each $z=0,1, \ldots, u-1$, we save the values of $d(t u, t u+z)$ in $W_{4}$. Because $A$ is an array of positive integers, we have $d(t u, t u+z) \leq z$ and $W_{4}$ needs at most $O\left(\left\lceil\frac{m}{u \log ^{2} u}\right\rceil u \log u\right)=o(m)$ bits. Otherwise, let

$$
W_{5}\left[A\left[\operatorname{index}_{\text {sum }}(A, t u), \text { index }_{\text {sum }}(A, t u)+\left\lceil\log ^{2} u\right\rceil-1\right]\right][z]=d(t u, t u+z)
$$

for each $z=0,1, \ldots, u-1 . W_{5}$ takes $O\left(2^{\log ^{3} u} u \log \log u\right)=o(m)$ bits and is obtainable in $O(m)$ time. Now, let $\alpha_{A}(A, m)=W_{3} \circ W_{4} \circ W_{5}$, which requires $o(m)$ bits and can be obtained in $O(m)$ time. To answer index sum $(A, x)$ in $O(1)$ time, first let $t$ and $z$ be the integers with $x=t u+z$ and $0 \leq z<u$, and then find the values of index $x_{\text {sum }}(A, t u)$ and $d(t u, t u+z)$ from $\alpha_{A}(A, m)$. The answer is index $_{\text {sum }}(A, t u)+d(t u, t u+z)$.

Let child_select $\left(S, v_{p}, c\right)$ denote the index $\ell_{k}$ such that $v_{k}$ is the $c$ th child of $v_{p}$. We have the following theorem.

THEOREM 4.6. It takes $O(n)$ time to compute an o(n)-bit string $\alpha_{\text {new } 3}$ such that child_select $\left(S, v_{p}, c\right)$ for each node $v_{p}$ and c can be answered from $S$ and $\alpha_{n e w}$ in $O$ (1) time.

Proof. We say that nodes in a set $D$ are $d$-disjoint [Chiang et al. 2005] if

- $w\left(\ell_{i}, r_{i}\right)>d$ holds for any node $v_{i}$ in $D$; and

- any two nodes $v_{i}$ and $v_{j}$ in $D$ satisfy at least one of $\left|w\left(\ell_{i}, \ell_{j}\right)\right|>d$ and $\left|w\left(r_{i}, r_{j}\right)\right|>d$.

Let $X$ be a $2\left\lceil\frac{2 n}{d}\right\rceil$-element array. For each $t=1,2, \ldots,\left\lceil\frac{2 n}{d}\right\rceil$, we store $v_{i}$ in $X[2 t-$ $1]$, where $\ell_{i}$ is the smallest index, if any, with $(t-1) d<\ell_{i} \leq t d$ such that $v_{i}$ is in $D$; and also store $v_{j}$ in $X[2 t]$, where $r_{j}$ is the largest index, if any, with $(t-1) d<r_{j} \leq t d$ such that $v_{j}$ is in $D$. Then, every node $v_{i}$ in $D$ takes at least one slot in $X$, and can be easily verified using $\ell_{i}$ and $r_{i}$. We simply say that $X$ has $v_{i}$ if and only if $v_{i}$ takes at least one of $X\left[2\left\lceil\frac{\ell_{i}}{d}\right\rceil-1\right]$ or $X\left[2\left\lceil\frac{r_{i}}{d}\right\rceil\right]$. For notational brevity, let $X\left[v_{i}\right]$ denote the element taken by $v_{i}$. 
The preprocessing is under the following traversal procedure: first traverse each node $v_{p}$ of $T$ in prefix order, and for each $v_{p}$ traverse every child $v_{i}$ of $v_{p}$ in counterclockwise order. Since selecting and matching a parenthesis on $S$ takes $O(1)$ time, and each node is traversed at most two times, one as $v_{p}$ and the other as $v_{i}$, the whole procedure takes $O(n)$ time. The discussion below focuses on nodes $v_{p}$ and $v_{i}$ in each iteration of the aforementioned traversal.

- Case 1. $v_{i}$ is a wide child of $v_{p}$. Let counter denote the number of wide nodes discovered before each iteration. It is not difficult to see that the parents of wide nodes are $B$-disjoint. Let $X_{1}$ be the $2 n_{B}$-element array with $X_{1}\left[v_{p}\right]=$ (before $_{p}$, first, last), where before $_{p}$ is the value of counter before we get $v_{p}$, and first (respectively, last) is the rank of the first (respectively, last) wide child of $v_{p}$. Then we partition $S$ into length- $B$ blocks. Let $Y_{1}$ be the $n_{B}$-element array with $Y_{1}[t]=\left(\right.$ before $\left._{i}, \ell_{i}\right)$, where $\ell_{i}$ is the smallest index in a block such that $v_{i}$ is wide, before $_{i}$ is the value of counter before we get $v_{i}$, and $t$ is the first empty entry of $Y_{1}$. Both of $X_{1}$ and $Y_{1}$ take $O\left(n_{B} \log n\right)=o(n)$ bits.

- Case 2. $v_{i}$ is a medium child of $v_{p}$. First, we partition $S$ into length- $B$ blocks. If $w\left(\ell_{p}, \ell_{i}\right) \leq B$, we say that $v_{i}$ belongs to the $\left\lceil\frac{\ell_{p}}{B}\right\rceil$-th block, otherwise the $\left\lceil\frac{r_{p}}{B}\right\rceil$-th block. For each $t=1,2, \ldots, n_{B}$, let counter $[t]$ denote the number of medium nodes belonging to the $t$ th block before each iteration. Note that at most $B$ medium nodes belong to a block. Similarly, one can verify that the parents of medium nodes are $b$-disjoint. Let $X_{2}$ be the $2 n_{b}$-element array with

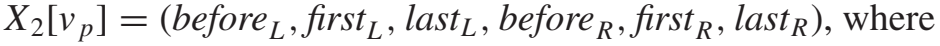

${\text { - } \text { before }_{L} \text { (respectively, before }}_{R}$ ) is the value of counter $\left[\left\lceil\frac{\ell_{p}}{B}\right\rceil\right]$ (respectively, the value of counter $\left.\left[\left\lceil\frac{r_{p}}{B}\right\rceil\right]\right)$ before we get $v_{p}$,

- first $_{L}$ (respectively, first ${ }_{R}$ ) is the rank of the first medium child of $v_{p}$ belonging to the $\left\lceil\frac{\ell_{p}}{B}\right\rceil$-th (respectively, $\left\lceil\frac{r_{p}}{B}\right\rceil$-th) block, and

- last $_{L}$ (respectively, last $t_{R}$ ) is the rank of the last medium child of $v_{p}$ belonging to the $\left\lceil\frac{\ell_{p}}{B}\right\rceil$-th (respectively, $\left\lceil\frac{r_{p}}{B}\right\rceil$-th) block.

Note that $1 \leq$ first $_{L} \leq$ last $_{L} \leq B$ and degree $\left(S, v_{p}\right)-B \leq$ first $_{R} \leq$ last $_{R} \leq$ degree $\left(S, v_{p}\right)$. We further partition each length- $B$ block into length- $b$ blocks. For each $t=1,2, \ldots, n_{B}$, let $Y_{2}[t]$ be the $\left\lceil\frac{B}{b}\right\rceil$-element array with $Y_{2}[t][u]=$ (before ${ }_{i}, \ell_{i}$ ), where $\ell_{i}$ is the smallest index in a length- $b$ block such that $v_{i}$ is a medium node belonging to the $t$ th length- $B$ block, before is the value of counter $[t]$ before we get $v_{k}$, and $u$ is the first empty entry of $Y_{2}[t]$. Observe that $X_{2}$ needs $O\left(n_{b} \log B\right)=o(n)$ bits and $Y_{2}$ needs $O\left(n_{B}\left\lceil\frac{B}{b}\right\rceil \log B\right)=o(n)$ bits.

For each $t=1,2, \ldots, n_{B}$, let $\alpha_{B 1}[t]=\alpha_{B}\left(\ell_{i}\right)$ with $\left(\right.$ before $\left._{i}, \ell_{i}\right)=Y_{1}[t]$. By Lemma 4.4, $\alpha_{B 1}$ takes $o(n)$ bits and is obtainable in $O(n)$ time. Let $A_{1}$ be the $n_{B}$-element array such that $\sum_{t=1}^{u} A_{1}[t]=$ before $_{i}$ with (before $\left._{i}, \ell_{i}\right)=Y_{1}[u]$ holds for each $u=1,2, \ldots, n_{B}$. Note that $0<A_{1}[t] \leq B$ holds for any index $t$, so $A_{1}$ takes $O\left(n_{B} \log B\right)=o(n)$ bits. Also, for each $t=1,2, \ldots, n_{B}$, let $A_{2}[t]$ be the $\left\lceil\frac{B}{b}\right.$ 7-element array such that $\sum_{u=1}^{x} A_{2}[t][u]=$ before $_{i}$ with (before $\left._{i}, \ell_{i}\right)=Y_{2}[t][x]$ holds for each $x=1,2, \ldots,\left\lceil\frac{B}{b}\right\rceil$. Observe that $0<A_{2}[t][u] \leq b$ holds for any indices $t$ and $u$, so $A_{2}$ takes $O\left(n_{B}\left\lceil\frac{B}{b}\right\rceil \log b\right)=o(n)$ bits. Let $\alpha_{A 1}=\alpha_{A}\left(A_{1}, n\right)$, and for each $t=1,2, \ldots, n_{B}$, let $\alpha_{A 2}[t]=\alpha_{A}\left(A_{2}[t], B\right)$. By Lemma 4.5, both of $\alpha_{A 1}$ and $\alpha_{A 2}$ take $o(n)$ bits and are obtainable in $O(n)$ time. At last, we construct an 


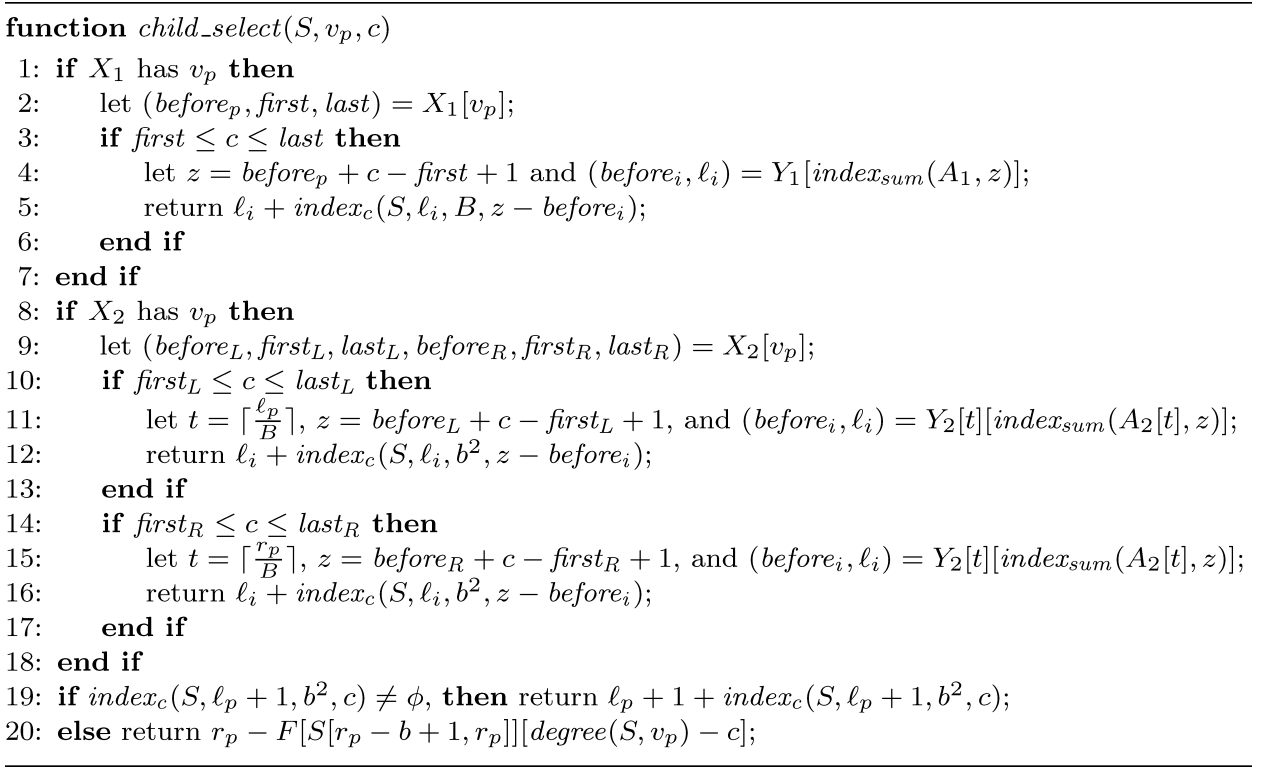

FIG. 3. An $O(1)$-time algorithm that computes $\operatorname{child} \_$select $\left(S, v_{p}, c\right)$.

$O(n)$-time obtainable table $F$ with $F\left[S\left[r_{p}-b+1, r_{p}\right]\right]\left[\operatorname{degree}\left(S, v_{p}\right)-c\right]=r_{p}-\ell_{i}$, where $v_{i}$ is the $c$ th child of $v_{p}$ with $w\left(\ell_{i}, r_{p}\right) \leq b$. Note that degree $\left(S, v_{p}\right)-c \leq b$, so $F$ takes $O\left(2^{b} b \log b\right)=o(n)$ bits.

To implement child select in $O(1)$ time, let $\ell_{k}=\operatorname{child} \_\operatorname{select}\left(S, v_{p}, c\right) . v_{k}$ is wide if and only if $X_{1}$ has $v_{p}$ and first $\leq c \leq$ last, where (before $_{p}$, first, last) $=X_{1}\left[v_{p}\right]$. Moreover, letting $z=$ before $_{p}+c-$ first $+1, v_{k}$ is the $z$ th wide node discovered during the traversal procedure. Let $\left(\right.$ before $\left._{i}, \ell_{i}\right)=Y_{1}\left[\operatorname{index}_{\text {sum }}\left(A_{1}, z\right)\right]$, so $v_{k}$ is a sibling of $v_{i}$ with $w\left(\ell_{i}, \ell_{k}\right) \leq B$ such that $\operatorname{child} \_r a n k\left(S, v_{k}\right)=\operatorname{child} \_r a n k\left(S, v_{i}\right)+$ $z$ - before $_{i}$. By Lemma 4.4, we can locate $v_{k}$ using $\ell_{k}=\ell_{i}+\operatorname{index}_{c}\left(S, \ell_{i}, B, z-\right.$ before $_{i}$ ).

$v_{k}$ is medium if and only if $X_{2}$ has $v_{p}$ and at least one of first $_{L} \leq c \leq$ last $_{L}$

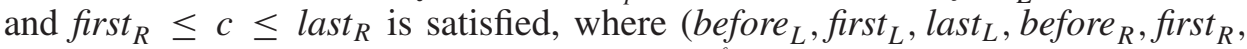
last $\left._{R}\right)=X_{2}\left[v_{p}\right]$. If first ${ }_{L} \leq c \leq$ last $_{L}$, let $t=\left\lceil\frac{\ell_{p}}{B}\right\rceil$ and $z=$ before $_{L}+c-$ first $_{L}+1$. If first $_{R} \leq c \leq$ last $_{R}$, let $t=\left\lceil\frac{r_{p}}{B}\right\rceil$ and $z=$ before $_{R}+c-$ first $_{R}+1$. Then, $v_{k}$ is the $z$ th medium node belonging to the $t$ th length- $B$ block discovered during the traversal procedure. Let before $\left._{i}, \ell_{i}\right)=Y_{2}[t]\left[\right.$ index $\left._{\text {sum }}\left(A_{2}[t], z\right)\right]$, so $v_{k}$ is a sibling of $v_{i}$ with $w\left(\ell_{i}, \ell_{k}\right) \leq b$ such that child_rank $\left(S, v_{k}\right)=\operatorname{child\_ rank}\left(S, v_{i}\right)+z-$ before $_{i}$. By Lemma 4.3, we can locate $v_{k}$ using $\ell_{k}=\ell_{i}+\operatorname{index}_{c}\left(S, \ell_{i}, b^{2}, z-\right.$ before $\left._{i}\right)$.

If $v_{k}$ is neither wide nor medium, it must be narrow. If $\operatorname{index}_{c}\left(S, \ell_{p}+1, b^{2}, c\right) \neq \phi$, then we have $\ell_{k}=\ell_{p}+1+\operatorname{index}_{c}\left(S, \ell_{p}+1, b^{2}, c\right)$. Otherwise, $\ell_{k}=r_{p}-F\left[S\left[r_{p}-\right.\right.$ $\left.\left.b+1, r_{p}\right]\right]\left[\operatorname{degree}\left(S, v_{p}\right)-c\right]$.

Finally, let $\alpha_{\text {new } 3}=\alpha_{\text {aux }} \circ \alpha_{b} \circ \alpha_{B 1} \circ X_{1} \circ Y_{1} \circ X_{2} \circ Y_{2} \circ A_{1} \circ \alpha_{A 1} \circ A_{2} \circ \alpha_{A 2} \circ F$, which takes $o(n)$ bits and can be computed from $S$ in $O(n)$ time. The $O(1)$-time algorithm for computing child_select $\left(S, v_{p}, c\right)$ is shown in Figure 3.

ACKNOWLEDGMENT. We thank Kai-min Chung for helpful discussion. We also thank the anonymous reviewers for their helpful comments. 


\section{REFERENCES}

Bell, T. C., Cleary, J. G., And Witten, I. H. 1990. Text Compression. Prentice-Hall, Englewood Cliffs, NJ.

Bender, M. A., And Farach-Colton, M. 2000. The LCA problem revisited. In Proceedings of the 4th Latin American Symposium on Theoretical Informatics (Punta del Este, Uruguay), G. H. Gonnet, D. Panario, and A. Viola, Eds. Lecture Notes in Computer Science, vol. 1776. Springer-Verlag, New York, 88-94.

Benoit, D., Demaine, E. D., MunRo, J. I., Raman, R., Raman, V., And RaO, S. S. 2005. Representing trees of higher degree. Algorithmica 43, 4, 275-292.

Bonichon, N., Gavollle, C., Hanusse, N., Poulalhon, D., And Schaeffer, G. 2006. Planar graphs, via well-orderly maps and trees. Graph and Combinat. 22, 1-18.

CHIANG, Y.-T., LIN, C.-C., AND LU, H.-I. 2005. Orderly spanning trees with applications. SIAM J. Comput. 34, 4, 924-945.

Chuang, R. C.-N., GARG, A., He, X., KAO, M.-Y., AND LU, H.-I. 1998. Compact encodings of planar graphs via canonical ordering and multiple parentheses. In Proceedings of the 25th International Colloquium on Automata, Languages, and Programming (Aalborg, Denmark), K. G. Larsen, S. Skyum, and G. Winskel, Eds. Lecture Notes in Computer Science, vol. 1443. Springer-Verlag, New York, 118-129.

ClARK, D. R. 1996. Compact PAT trees. Ph.D. dissertation, University of Waterloo.

Clark, D. R., AND MunRo, J. I. 1996. Efficient suffix trees on secondary storage. In Proceedings of the 7th Annual ACM-SIAM Symposium on Discrete Algorithms (Atlanta, CA). ACM, New York, 383-391.

DelPRATT, O., RAHMAN, N., AND RAMAN, R. 2006. Engineering the LOUDS succinct tree representation. In Proceedings of the 5th International Workshop on Experimental Algorithms (Cala Galdana, Menorca, Spain), Lecture Notes in Computer Science, vol. 4007. Springer-Verlag, New York, 134145.

ELIAS, P. 1975. Universal codeword sets and representations of the integers. IEEE Trans. Inf. Theory IT21, 194-203.

Gabow, H. N., Bentley, J. L., AND TARJAN, R. E. 1984. Scaling and related techniques for geometry problems. In Proceedings of the 16th Annual ACM Symposium on Theory of Computing. ACM, New York, 135-143.

GEARY, R. F., RAHMAN, N., RAMAN, R., AND RAMAN, V. 2004a. A simple optimal representation for balanced parentheses. In Proceedings of the 15th Annual Symposium on Combinatorial Pattern Matching (Istanbul, Turkey), S. C. Sahinalp, S. Muthukrishnan, and U. Dogrusöz, Eds. Lecture Notes in Computer Science, vol. 3109. Springer-Verlag, New York. 159-172.

GeARY, R. F., RAman, R., AND RAman, V. 2004b. Succinct ordinal trees with level-ancestor queries. In Proceedings of the Fifteenth Annual ACM-SIAM Symposium on Discrete Algorithms (New Orleans, LA), J. I. Munro, Ed. ACM, New York, 1-10.

Graham, R. L., Knuth, D. E., And Patashnik, O. 1989. Concrete Mathematics. Addison-Wesley, Reading, MA.

HE, X., KAO, M.-Y., AND LU, H.-I. 1999. Linear-time succinct encodings of planar graphs via canonical orderings. SIAM J. Disc. Math. 12, 3, 317-325.

Hon, W.-K., SADAKANE, K., AND SunG, W.-K. 2003. Succinct data structures for searchable partial sums. In Proceedings of the 14th Symposium on Algorithms and Computation (Kyoto, Japan), T. Ibaraki, N. Katoh, and H. Ono, Eds. Lecture Notes in Computer Science, vol. 2906. Springer-Verlag, New York, 505-516.

JACOBSON, G. 1989. Space-efficient static trees and graphs. In Proceedings of the 30th Annual Symposium on Foundations of Computer Science (Research Triangle Park, NC). IEEE Computer Society Press, Los Alamitos, CA, 549-554.

MunRo, J. I. 1996. Tables. In Proceedings of the 16th Conference on Foundations of Software Technology and Theoretical Computer Science (Hyderabad, India), Lecture Notes in Computer Science, vol. 1180. Springer-Verlag, New York, 37-42.

MunRO, J. I., AND RAMAN, V. 2001. Succinct representation of balanced parentheses, static trees and planar graphs. SIAM J. Comput. 31, 3, 762-776.

MunRo, J. I., Raman V., AND RaO, S. S. 2001. Space efficient suffix trees. J. Algor. 39, 2, $205-$ 222.

MunRo, J. I., AND RaO, S. S. 2004. Succinct representations of functions. In Proceedings of the 31st International Colloquium on Automata, Languages and Programming (Turku, Finland), Lecture Notes in Computer Science, vol. 3142. Springer-Verlag, New York, 1006-1015. 
RAman, R., Raman, V., And RaO, S. S. 2001. Succinct dynamic data structures. In Proceedings of the 7th International Workshop on Algorithms and Data Structures (Providence, RI), F. K. H. A. Dehne, J.-R. Sack, and R. Tamassia, Eds. Lecture Notes in Computer Science, vol. 2125. Springer-Verlag, New York, 426-437.

SADAKANE, K. 2002. Succinct representations of LCP information and improvements in the compressed suffix arrays. In Proceedings of the 13th Annual ACM-SIAM Symposium on Discrete Algorithms, (San Francisco, CA). ACM, New York, 225-232.

VAN EMDE BOAS, P. 1990. Machine models and simulations. In Handbook of Theoretical Computer Science, J. van Leeuwen, Ed. Vol. A. Elsevier, Amsterdam, The Netherlands, Chapter 1, 1-60.

RECEIVED DECEMBER 2005; REVISED OCTOBER 2006 AND MARCH 2007; ACCEPTED APRIL 2007 